Mathematical Research Letters 4, 889-894 (1997)

\title{
PERCOLATION ON THE PROJECTIVE PLANE
}

\author{
Michael H. Freedman
}

\begin{abstract}
Since the projective plane is closed, the natural homological observable of a percolation process is the presence of the essential cycle in $H_{1}\left(R P^{2} ; Z_{2}\right)$. In the Voroni model at critical phase, $p_{c}=.5$, this observable has probability $q=.5$ independent of the metric on $R P^{2}$. This establishes a single instance $\left(R P^{2}\right.$, homological observable) of a very general conjecture about the conformal invariance of percolation due to Aizenman and Langlands, for which there is much moral and numerical evidence but no previously verified instances. On $R P^{2}$ all metrics are conformally equivalent so the proof of metric independence is precisely what the conjecture would predict. What is very special, is that at $p_{c}$ metric invariance holds in all finite models so passing to the limit is trivial; the probability $q$ is fixed at .5 by a topological symmetry.
\end{abstract}

Percolation is the effect, at larger size scales, of randomly laying down material at a small scale. The arena may be a surface with or without boundary or a higher dimensional manifold. Although the continuum limit is the most interesting regime, to date it has been necessary to approach this as a limit of a discrete problem. Many related discrete forms have been studied: (1) lattice models in which bonds (or vertices) are "chosen" at random, (2) lilly pad models in which metric disks about randomly selected points are chosen, and (3) Voronoi models in which Voronoi domains about randomly selected capitals are themselves chosen independently with probability, $p \in(0,1)$, [VW] and [BS]. We will think of the manifold as white and the chosen material black. The subject studies the statistical/geometric properties of the black material and its appearance in the high refinement or the "continuum limit". At the largest size scale in the problem we come to homological questions: Classically it is asked what is the probability of finding a black path (i.e. a path in the black region) across a 1-connected planar domain $D$ with endpoints lying on fixed disjoint sub-arcs $\subset \partial D$. This amounts to asking the probability that the nontrivial element of $H_{1}\left(D, \operatorname{arc}_{1} \cup \operatorname{arc} 2\right)$ comes from the inclusion of the black subset, $B$. Similarly on a closed surface $S$ it is natural to study the probability that any given absolute element $\in H_{1}(S)$ is in the image of $B$. These elements are "homological observables" of the process and their expected values are of interest. Further generalizing, given a percolation process on a manifold $M$, the event that an absolute (or relative) homology class comes from $B$ is "homological observable". Such observables live at the largest scale.

Received September 9, 1997. 
It is a remarkable conjecture of Michael Aizenman based partly on computer studies of Langlands et al. (see [L]), and partly on connections with conformal fields theory, which were further developed by J. Cardy $[\mathrm{C}]$, that for surfaces, at the critical phase ${ }^{1} p_{c}$ the entire percolation processes in the continuum limit depends only on the conformal structure (not the entire metric). Theorem 1 below verifies conformal invariance of the single homological observable for the real projective plane, $R P^{2}$. Certain generalizations are given in Theorem 2 and Propositions 1, 2, 3, and 4 .

The projective plane is a very special case for percolation because: (1) there is exactly one essential homology class or "observable" in the middle dimension, and (2) the projective plane (like the sphere) is conformally unique - any two Riemannian metrics on $R P^{2}$ admit a conformal isomorphism. In view of the latter, "conformal invariance" for percolation on $R P^{2}$ actually means "metric invariance". We prove that in a discrete setting which generalizes the Voronoi model that "metric invariance" holds exactly at $p_{c}=.5$; it is not necessary to pass to any limit.

For a surface $S$, the discrete notion of metric that we use is a "generic cellulation". That is $S$ is tiled by finitely-many closed piecewise-smooth 2-cells with disjoint interiors so that no more than three meet at any point. Such a cellulation might arise in the Voronoi model given a metric and a Poisson process but we need no such assumption. Conversely one may think of a cellulation itself as a discrete approximation to a metric. The two-fold incidence relations of the tiling can be realized using Andreev's theorem ([T] and $[\mathrm{RS}])$ as a disk packing on a unique surface $S^{\prime}$ of constant curvature (the projective plane in the case of Theorem 1 below). This surface $S^{\prime}$ together with the coarse map $S \rightarrow S^{\prime}$ determined by: cells $\rightarrow$ disks is the induced geometric structure.

Lemma 1. Let $C$ be a generic cellulation of $R P^{2}$ with $n$ cells. Of the $2^{n}$ possible black-white colorings of $C$, exactly $2^{(n-1)}$ admit an essential loop lying entirely in the black region.

Proof. By genericity of $C$ the black $(B)$ and white $(W)$ subsets are closed subsurfaces meeting along their boundaries. We claim that exactly one of $B$ and $W$ contains an essential loop in $R P^{2}$. Every boundary circle $\gamma$ between $B$ and $W$ is two-sided and therefore bounds a disk in $R P^{2}$. To see this, note that if $\gamma$ bounded a non-disk on both sides, each of these sides would have nonpositive Euler-characteristic and by additivity we would obtain the contradiction $\chi\left(R P^{2}\right) \leq 0$. It follows that $R P^{1}$ contained in $P R^{2}$ can be isotoped (i.e. pushed) off all these circles one at a time and therefore into either $B$ or $W$; thus at least one side contains an essential loop - that is a loop which carries the non-zero element of $H_{1}\left(R P^{2}, Z_{2}\right)$. Because the $Z_{2^{-}}$intersection form on $R P^{2}$ is onedimensional $\langle 1\rangle$ any two essential loops must have intersection number equal one. Thus it is impossible for both $B$ and $W$ to contain essential loops, for these

\footnotetext{
${ }^{1}$ In the 2-dimensional Voronoi model and on self-dual lattice models $P_{c}=.5$ by symmetry considerations.
} 
could be isotoped into the respective interiors where they would necessarily be disjoint. This proves the claim. There is a fixed-point free symmetry of the colorings of $C$ which for any given coloring interchanges $B$ and $W$. In view of the claim, the condition that there be an essential loop in the black side $B$ defines a section of the quotient map $\pi$ : colorings of $C \rightarrow$ colorings of $C /$ involution. Thus the number of colorings with an essential black loop is the cardinality of the quotient space $2^{(n-1)}$.

From the preceding discussion and the Lemma we have:

Theorem 1. In the Voronoi model at any level of refinement and at $p_{c}=.5$, the (unique) homological observable on $\left(R P^{2}\right)$ i.e. the event "the generator of $H_{1}\left(R P^{2} ; Z_{2}\right)$ lies in black" is conformally (equivalently metric) invariant. It occurs with probability $q=.5$ independent of the metric on $R P^{2}$.

The "torus trick" of Kirby [K] and later Sullivan [S] show that topological and geometric problems can sometimes be profitably localized onto a closed manifold. In this spirit we suggest that the duality on the projective plane might figure into a proof of the general conjecture. Much of the progress in two-dimensional percolation rests on the metrical symmetry of the square. The symmetry of the projective plane is inherently topological and therefore more robust.

We now consider closed manifolds of dimension $\geq 2$. Let $\mathcal{C}$ be a class of closed manifolds (possibly a singleton) and $P(B, W)$ is a property of decompositions $B \cup W=M \in \mathcal{C}$, where $B$ and $W$ are submanifolds of $M$ meeting along their common boundary, taking values in $Z_{2}=\{$ true, false $\}$. We call $P$ an exclusive property if exactly one of $P(B, W)$ and $P(W, B)$ is true.

Let $M$ be a closed smooth manifold of with a compatible P.L. triangulation. We consider the process which colors the dual cells to this triangulation black with probability $=.5$. We may imagine that the cells are generated from a metric (and its associated volume form) by selecting "capitals" by a Poisson process and expanding these to "countries" or Voronoi cells. Generically such cells will be dual to a compatible P.L. triangulation. For the topological discussion it will not matter how these cells are generated only that any union of them is a piece-wise smooth, codimension zero, submanifold with boundary.

An immediate generalization of Theorem 1 is:

Theorem 2. Let $\mathcal{C}$ be some class of manifolds for which an exclusive property $P$ is defined. In the Voronoi model of percolation, at any level of refinement, and at $p_{c}=.5$, the observable on $M=B \cup W, " P(B, W)$ is true" occurs with probability $=.5$ independent of the metric on $M$.

We establish four (related) exclusive properties; readers are encouraged to find more.

Let $S_{n}$ be the connected sum of $2 n+1$ copies of $R P^{2}, \quad\left\{S_{n}, n \geq 0\right\}$ is our class $\mathcal{C}_{1}$. And for any decomposition of $S_{n}$ into a black and white subsurfaces meeting along their common boundary, $S_{n}=B \cup W$ let $P_{1}$ be the property that: $\operatorname{dim}\left(\right.$ image $\left.H_{1}\left(B ; Z_{2}\right) \rightarrow H_{1}\left(S_{n} ; Z_{2}\right)\right)>\operatorname{dim}\left(\right.$ image $\left.H_{1}\left(W ; Z_{2}\right) \rightarrow H_{1}\left(S_{n} ; Z_{2}\right)\right)$. 
Proposition 1. $P_{1}$ is an exclusive property on $\mathcal{C}_{1}$.

Proof. Let $C$ be the 1-manifold $B \cap W$. The image $\left(H_{1}\left(C ; Z_{2}\right)\right)=I \subset H_{1}\left(S_{n} ; Z_{2}\right)$ is an isotropic subspace and its perpendicular space $I^{\perp}$ is easily identified as the image $\left(H_{1}\left(B, Z_{2}\right) \oplus H_{1}\left(W ; Z_{2}\right)\right) \stackrel{\text { inc }_{*} \oplus \text { inc }_{*}}{\longrightarrow} H_{1}\left(S_{n} ; Z_{2}\right)$. Writing the intersection form on $S_{n}$ as

$$
<,>=\bigoplus_{n} \begin{array}{c|cc}
\alpha_{i} & \beta_{i} \\
\beta_{i} & 0 & 1 \\
& 1 & 0
\end{array}|\oplus| 1 \mid
$$

an arbitrary $k$-dimensional isotropic subspace, $k \leq n$, may be mapped by an isomorphism of the intersection form onto the span of $\alpha_{i}, \ldots, \alpha_{k}$ within the maximal $n$-dimensional isotropic subspace, $\operatorname{span}\left(\alpha_{1}, \ldots, \alpha_{n}\right)$. In these coordinates, $I^{\perp}$ is spanned by

$$
\alpha_{1}, \ldots, \alpha_{k}, \alpha_{k+1}, \beta_{k+1}, \ldots, \alpha_{n}, \beta_{n}, \delta
$$

so $\operatorname{dim}\left(I^{\perp} / I\right)$ is odd. However

$$
\begin{aligned}
I^{\perp} / I & \cong \frac{\text { image } H_{1}\left(B ; Z_{2}\right) \oplus H_{1}\left(W ; Z_{2}\right) \subset H_{1}\left(S_{n} ; Z_{2}\right)}{\text { image } H_{1}\left(C ; Z_{2}\right) \subset H\left(S_{n} ; Z_{2}\right)} \\
& \cong \frac{\text { image } H_{1}\left(B ; Z_{2}\right) \oplus H_{1}\left(W ; Z_{2}\right) \subset H_{1}\left(S_{n} ; Z_{2}\right)}{\operatorname{image}\left(H_{1}\left(B ; Z_{2}\right) \subset H_{1}\left(S_{n} ; Z_{2}\right)\right) \cap \operatorname{image}\left(H_{1}\left(W ; Z_{2}\right) \subset H_{1}\left(S_{n} ; Z_{2}\right)\right)} .
\end{aligned}
$$

Setting $b, w$, and $i$ equal to the dimensions of the image of $H_{1}\left(B ; Z_{2}\right)$, the image of $H_{1}\left(B ; Z_{2}\right)$, and their intersection, respectively, we have: odd $=(b+w-i)-i$. Consequently $b=\operatorname{dim}$ (image $H_{1}\left(B ; Z_{2}\right) \subset H_{1}\left(S_{n} ; Z_{2}\right)$ ) is never equal to $w=$ $\operatorname{dim}\left(\right.$ image $H_{1}\left(W ; Z_{2}\right) \subset H_{1}\left(S_{n} ; Z_{2}\right)$ ).

We choose some coefficient field $F$ and restrict to the class of manifolds $\mathcal{C}_{2}$ with $\operatorname{dim}(M)=2 n$ and $H_{n}(M ; F) \cong F$, i.e. the middle dimensional homology has rank one. Now consider the property $P_{2}$ :

"The $\operatorname{map} H_{n}(B ; F) \rightarrow H_{n}(M ; F)$ is onto".

Proposition 2. $P_{2}$ is an exclusive property on $\mathcal{C}_{2}$.

Proof. Consider the Mayer-Vietovis sequence with coefficients in F.

$$
\begin{aligned}
& \rightarrow H_{n}(B \cap W) \rightarrow H_{n}(B) \oplus H_{n}(W) \rightarrow H_{n}(M) \stackrel{\partial}{\longrightarrow} H_{n-1}(B \cap W) \rightarrow \\
& \begin{array}{ccc}
\Psi \alpha & \Psi & \Psi \\
\partial \alpha & \alpha & \partial \alpha
\end{array}
\end{aligned}
$$

Let $\alpha$ denote a generator. The $\partial$-map is given by transverse intersection. In the homology of the $n$-1-manifold $B \cap W, \widehat{\partial \alpha}$ denotes a Poincaré dual to $\partial \alpha$ which exists unless $\partial \alpha=0$. The intersection number $\left\langle\widehat{\partial \alpha}, \partial \alpha>_{(B \cap W)}=1 \in F\right.$. Since the normal bundle $\nu_{B \cap W \subset M}$ is trivial ("toward $B$ " is a section) the intersection 
number $<\widehat{\partial \alpha}, \widehat{\partial \alpha}>_{M}=0 \in F$. Thus by our hypothesis on $M$, inc $_{*} \widehat{\partial \alpha}=0 \in$ $H_{n}(M)$ otherwise the one dimensional intersection form on $M$ would be singular contradicting Poincaré duality. But

$$
<\widehat{\partial \alpha}, \partial \alpha>_{(B \cap W)}=<\widehat{\partial \alpha}, \alpha>_{M}=<0, \alpha>_{M}=0 .
$$

This contradiction implies $\partial \alpha=0$. Using the exactness of the Mayer-Vietovis sequence and (again) the one dimensionality of $H_{n}(M)$ we see that at least one of the inclusions $H_{n}(B) \rightarrow H_{n}(M)$ or $H_{n}(W) \rightarrow H_{n}(M)$ must be an epimorphism. As in the proof of Lemma 1, only one of these inclusions can be an epimorphism, otherwise a cycle with non-zero self intersection could be represented in two disjoint regions, interior $B$ and interior $W$.

Let $\mathcal{C}_{3}$ be the class of closed smooth $4 k$-dimensional manifolds with odd signature and for $B \cup W=M \in \mathcal{C}_{3}$ let $P_{3}(B, W)$ state "signature $B>$ signature W".

Proposition 3. $P_{3}$ is an exclusive property on $\mathcal{C}_{3}$.

Proof. The proposition follows directly from Novikoff's additivity property for signature (see $[\mathrm{W}])$.

Note that both Propositions 2 and 3 apply to the well known manifolds $C P^{2}$ and $H P^{2}$.

A final example is less interesting for percolation since it depends on the details of the subset, for which there is no bound on complexity, rather than upon its homological image. Let $\mathcal{C}_{4}$ be the class of closed manifolds with odd Euler characteristic $=\mathcal{X}$, and for $B \cup W=M \in \mathcal{C}_{4}$, let $P_{4}(B, W)$ state " $\mathcal{X}(B)>$ $\mathcal{X}(W)^{\prime \prime}$.

Proposition 4. $P_{4}$ is an exclusive property on $C_{4}$.

Proof. Euler characteristic is additive.

Using the axiom of choice, rather absurd exclusive properties can be constructed on any manifold.

The four examples above are all isotopy invariant, meaning $P(B, W) \Longleftrightarrow$ $P\left(\mathcal{I}_{t} B, \mathcal{I}_{t} W\right)$, where $\mathcal{I}_{t}: M \times[0,1] \rightarrow M$ is an isotopy, $M=B \cup W$.

Theorem 3. No closed odd-dimenisional manifold has an exclusive isotopy invariant property. (The statement may be interpreted in the smooth, P.L., or topological categories.)

Proof. Every closed $(2 n+1)$-dimensional manifold $M^{2 n+1}$ may be described as an "open book", that is as a relative mapping cylinder of an automorphism $h:\left(N^{2 n}, \partial N^{2 n}\right) \rightarrow\left(N^{2 n}, \partial N^{2 n}\right)$, for some $2 n$-dimensional manifold $\mathrm{N}$, where $\left.h\right|_{\partial N^{2 n}}=i d_{\partial N^{2 n}}$ : This theorem is trivial for $n=0$, and proved in $[\mathrm{A}]$ for $n=1$ and [Law], [Q] for $n \geq 2$.

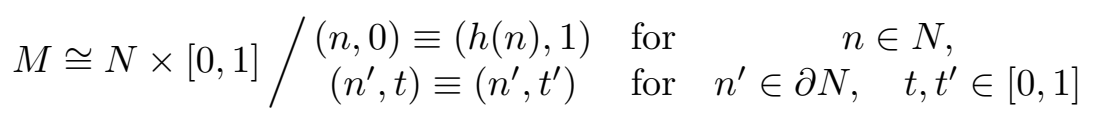


Setting $B=\operatorname{image}\left(N \times\left[0, \frac{1}{2}\right]\right)$ and $W=\operatorname{image}\left(N \times\left[\frac{1}{2}, 1\right]\right) \subset M$, we see that the isotopy of $\mathrm{M}$ :

$$
\mathcal{I}_{t}(n, s)=\begin{array}{cc}
(n, s+t) & s+t \leq 1 \\
(h(n), s+t-1) & s+t \geq 1,
\end{array} \quad s \in[0,1], t \in\left[0, \frac{1}{2}\right], \text { and } n \in N
$$

exchanges the subsets $B$ and $W$ so no isotopy-invariant exclusive property can exist.

A different proof of Theorem 3 using Cerf theory has been found by Peter Teichner (personal communication); he also points out that the Kirby-Siebenmann invariant yields an exclusive property on the class of closed, nonstably-smoothable, four-dimensional topological manifolds. We wonder if it is impossible for a closed manifold of even Euler characteristic to admit a diffeomorphism-invariant exclusive property.

\section{Acknowledgements}

I would like to thank Jennifer Chayes and Christian Borgs for introducing me to percolation.

\section{References}

[A] J. W. Alexander, A lemma on systems of knotted curves, Proc. Natn. Acad. Sci. 9 (1923), 93-95.

[BS] Benjamini and O. Schramm, Conformal invariance of Voronoŭ percolation (to appear).

[C] J. Cardy, Critical percolation in finite geometries, J. Phys. A 25 (1992), L201-L206.

[K] R. Kirby, Stable homeomorphisms and the annulus conjecture, Ann. of Math. 89 (1969), 575-582.

[L] R. Langlands, P. Pouliot, and Y. Saint-Aubin, Conformal invariance in 2-dimensional percolation, Bull. Amer. Math. Soc. 30 (1994), 1-61.

[Law] T. Lawson, Open book decompositions for odd dimensional manifolds, Topology 17 (1978), 189-192.

[T] W. Thurston, Princeton Notes (1978).

[Q] F. Quinn, Open book decompositions, and the bordism of automorphisms, Topology 18 (1979), 53-73.

[S] D. Sullivan, Hyperbolic geometry and homeomorphisms, Proc. Georgia Topology Conf., Athens, GA 1977, Geometric topology, Academic Press, New York-London, 1979, pp. $543-555$.

[RS] B. Rodin and D. Sullivan, The convergence of circle packings to the Riemann mapping, J. Diff. Geom. 26 (1987), 349-360.

[VW] M. Q. Vahidi-Asl and J. C. Wierman, First-passage percolation on Voronou tessellation and Delaunay triangulation, Random Graphs '87 (Poznań, 1987), Wiley, Chichester, 1990, pp. 341-359.

[W] C. T. C. Wall, Non-additivity of the signature, Invent. Math. 7 (1969), 269-274.

Department of Mathematics, Univeristy of California, La Jolla, 9500 Gilman

Drive, La Jolla, CA 92093-0112

E-mail address: mfreedman@ucsd.edu 